\title{
THERMONUCLEAR RUNAWAYS ON ACCRETING HOT WHITE DWARFS
}

\author{
Dina Prialnik \\ Racah Institute of Physics, Hebrew University \\ Jerusalem, Israel \\ Oded Regev \\ Department of Astronomy, Columbia University \\ New York, NY 10027, U.S.A.
}

\begin{abstract}
Preliminary results of 1-D fully hydrodynamic numerical calculations including accretion and mass loss on a $1 M_{\odot}$ hot white dwarf are presented. For the case of $\dot{M}=10^{-8} M_{\odot} / \mathrm{yr}$ and $T_{\text {eff }}=125000^{\circ} \mathrm{K}$ we get flashes of duration of $\sim 30-40 \mathrm{yrs}$ which reccur every $\sim 1500$ years. During each flash $\sim 6 \times 10^{-6} M_{\odot}$ is lost $(\sim 40 \%$ of the accreted envelope.
\end{abstract}

Previous work on thermonuclear runaways on hot white dwarfs suggested their applicability to symbiotic stars. See e.g. Paczyński \& Rudak (1980), Iben (1982), Kenyon and Truran (1983). None of these works are both hydrodynamic and include accretion.

We use the method and computer code described in Prialnik (1986) to follow hydrodynamically the accretion and subsequent evolution of the outburst, including the fate of the envelope. In the project we intend to survey the $\dot{M}-T_{\text {eff }}$ parameter space systematically to find the regions of steady burning, nondegenerate flashes and degenerate flashes. Of interest is also the amount of mass lost in these events and their temporal characteristics.

Here we report the results of one case $\left(\dot{M}=10^{-8} M_{\odot} / \mathrm{yr}, T_{\text {eff }}=125000^{\circ} \mathrm{K}\right)$. The results are presented in the figures. In figures 1 and 2 the luminosity and the nuclear luminosity respectively is shown as a function of time. Two flashes with an interval of $\sim 1500$ years between them are displayed. The structure of a flash is shown in figures 3 and 4. Figure 3 shows the typical behavior of the luminosity (full curve) and the nuclear luminosity (dashed curve) as a function of time during a flash and in figure 4 the same is shown with regard to the effective temeprature $-T_{\text {eff }}$.

These results are similar to previous work (e.g. Iben 1982). During each flash we have computed, a mass of $\sim 6 \times 10^{-6} M_{\odot}$ was lost from the system (achieved escape velocity and was removed from the calculation).

We intend to continue these calculation also for other values of $M$ and $T_{\text {eff }}$.

REFERENCES: 1. Paczyński, B. and Rudak; 1980, Astr. Astrophys., 82, 349.

2. Iben, I., 1982, Astrophys. J., 259, 244.

3. Kenyon, S.J. and J.W. Truran, 1983, Astrophys. J., 273, 280.

4. Prialnik, D., 1986, Astrophys. J., 310, 1986. 


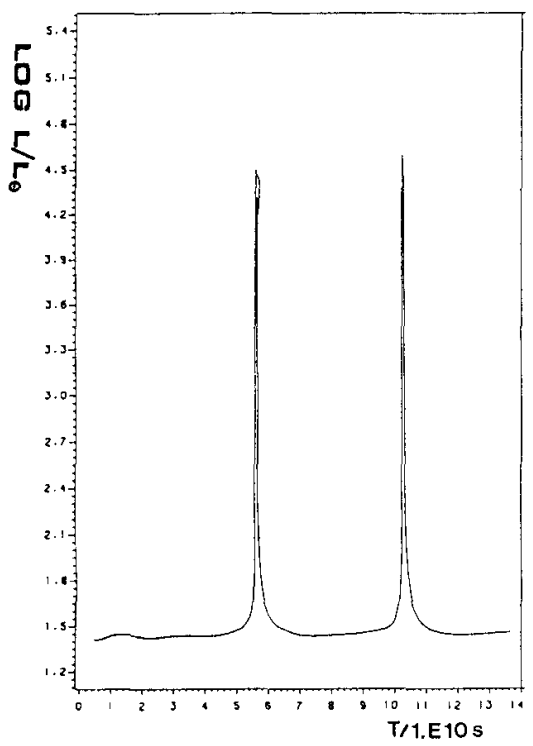

Figure 1 Luminosity as function of time

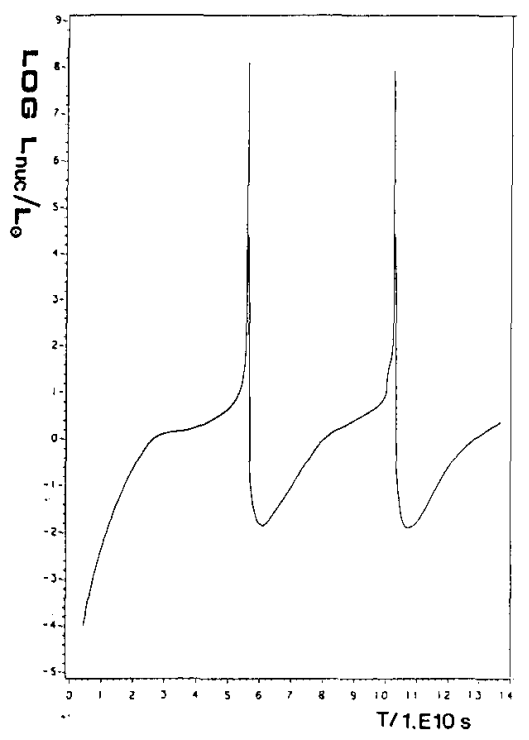

Figure 2

Nuclear luminosity as function of time

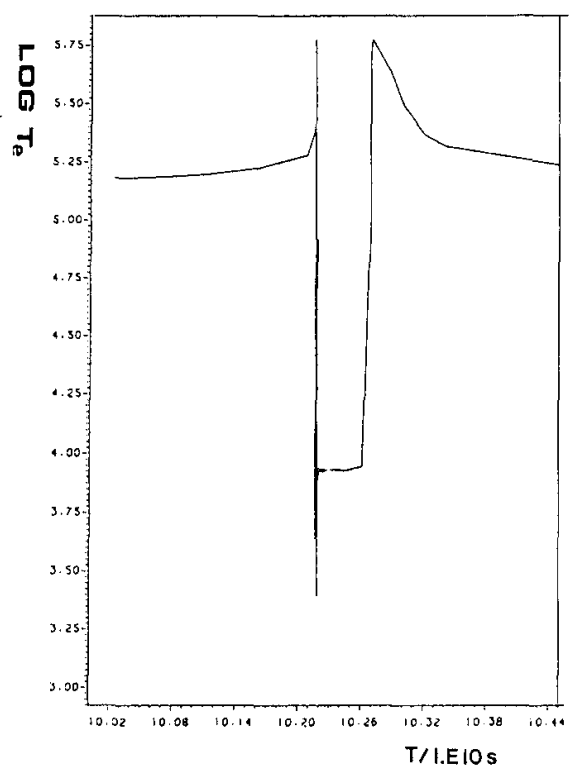

Figure 4

$T_{\text {eff }}$ as function of time 